\title{
Numerical analysis of a one-dimensional elastodynamic model of dry friction and unilateral contact
}

\author{
Y. Renard \\ SIGMAS Project, Laboratoire de Modélisation et Calcul LMC-IMAG, BP 53X, F-38041 Grenoble, France
}

\begin{abstract}
This paper deals with a numerical analysis of a one-dimensional dynamic purely elastic (i.e. hyperbolic) model with dry friction. Since we consider a Coulomb friction law with a slip velocity dependent coefficient, generally, the problem has more than one solution. A mass perturbation approach is developed to regain the uniqueness and to perform the numerical analysis. This study can be viewed as a first step in the numerical analysis of more elaborated dynamic purely elastic problems with dry friction.
\end{abstract}

\section{Introduction}

Friction laws with a slip velocity dependent coefficient were introduced to modelize the stick-slip phenomenon, which is the appearance of self-sustained vibration in mechanical systems submitted to dry friction. Even though there is not a universally accepted model of this phenomenon, the dynamic aspect seems to be essential in the behavior of such systems. Some mathematical and numerical results tend to prove that multi-dimensional systems submitted to dry friction and unilateral contact can develop instabilities even if the simplest Coulomb law with a constant coefficient is chosen (see $[3,15,11,16]$ ). Because of the results on systems with finite number of degree of freedom, the Coulomb law with a slip velocity dependent coefficient is still considered as a good candidate for the modelization of stick-slip motion of elastic structures.

The model described above is a one-dimensional purely elastic model with dry friction and unilateral contact. It is well known that there are major mathematical difficulties to treat such dynamical model, due to the bad regularity of the solutions (discontinuities in velocity). This work is a continuation of a paper [19] which treats the modeling part and also of the work of Ionescu and Paumier [8]. This onedimensional model, because of its simplicity, allows to enhance some fundamental properties of the dynamic behavior of elastic structure under dry friction and unilateral contact. The purpose here is to perform a numerical analysis of the purely elastic case, with a mass perturbation approach. This mass perturbation approach is also studied in [19] where it is proven, under certain restrictions, that the sequence of solutions of the perturbed problem converges toward a particular solution of the non-perturbed problem. This particular solution can be related to the selection made by the perfect delay criterion (see $[8,10])$. 


\section{The one-dimensional elastic model with dry friction}

The model we deal with in this paper is a slight extension of the one introduced in $[8,19]$, and was also studied in [17] and fully described in [18]. This model represents the dynamic motion of an elastic slab which slides with friction on a moving rigid foundation (see Fig. 1). The slab is assumed to be linearly elastic with constant Lamé coefficients $\lambda$ and $G$, density $\rho$ and height $H$.

With convenient initial data and lateral boundary conditions, it is possible to consider motions of the slab which only depend on the vertical coordinate $x_{3}$. So, if we denote by $\underline{u}\left(t, x_{3}\right)=\left(u_{1}\left(t, x_{3}\right)\right.$, $\left.u_{2}\left(t, x_{3}\right), u_{3}\left(t, x_{3}\right)\right)$ the displacement of the slab, the evolution of the vertical displacement $u_{3}\left(t, x_{3}\right)$ is described by the equation

$$
\left.\left.\left.\partial_{t t}^{2} u_{3}\left(t, x_{3}\right)=c_{2} \partial_{x_{3} x_{3}}^{2} u_{3}\left(t, x_{3}\right) \text { in }\right] 0, T\right] \times\right] 0, H[,
$$

where $c_{2}=\sqrt{(\lambda+2 G) / \rho}$ is the velocity of longitudinal waves. The slab is assumed to be fixed on its top

$$
\left.\left.u_{3}(t, H)=-D \quad \text { with } D>0 \forall t \in\right] 0, T\right],
$$

and it is submitted to a unilateral contact condition on $x_{3}=0$ :

$$
\left.\left.\partial_{x_{3}} u_{3}(t, 0) \leqslant 0, \quad-u_{3}(t, 0) \leqslant 0, \quad \partial_{x_{3}} u_{3}(t, 0) u_{3}(t, 0)=0 \quad \forall t \in\right] 0, T\right] .
$$

It is convenient to express this later condition by the inclusion

$$
\left.\left.(\lambda+2 G) \partial_{x_{3}} u_{3}(t, 0) \in-J_{N}\left(-u_{3}(t, 0)\right) \quad \forall t \in\right] 0, T\right],
$$

where the multi-valued map

$$
J_{N}(x)= \begin{cases}\{0\} & \text { if } x<0, \\ {[0,+\infty[} & \text { if } x=0,\end{cases}
$$

is the sub-differential of the convex indicator function of the interval $]-\infty, 0]$.

The evolution of the horizontal displacement $u_{T}\left(t, x_{3}\right)=\left(u_{1}\left(t, x_{3}\right), u_{2}\left(t, x_{3}\right)\right)$ is described by the equation

$$
\left.\left.\left.\partial_{t t}^{2} u_{T}\left(t, x_{3}\right)=c_{1} \partial_{x_{3} x_{3}}^{2} u_{T}\left(t, x_{3}\right), \quad \text { in }\right] 0, T\right] \times\right] 0, H[\text {, }
$$

where $c_{1}=\sqrt{G / \rho}$ is the velocity of transversal waves. On $x_{3}=H$ it is assumed that the slab is fixed

$$
\left.\left.u_{T}(t, H)=0 \quad \forall t \in\right] 0, T\right] .
$$

The friction is modelized by a Coulomb law of friction with a slip velocity dependent coefficient on the contact boundary $x_{3}=0$ :

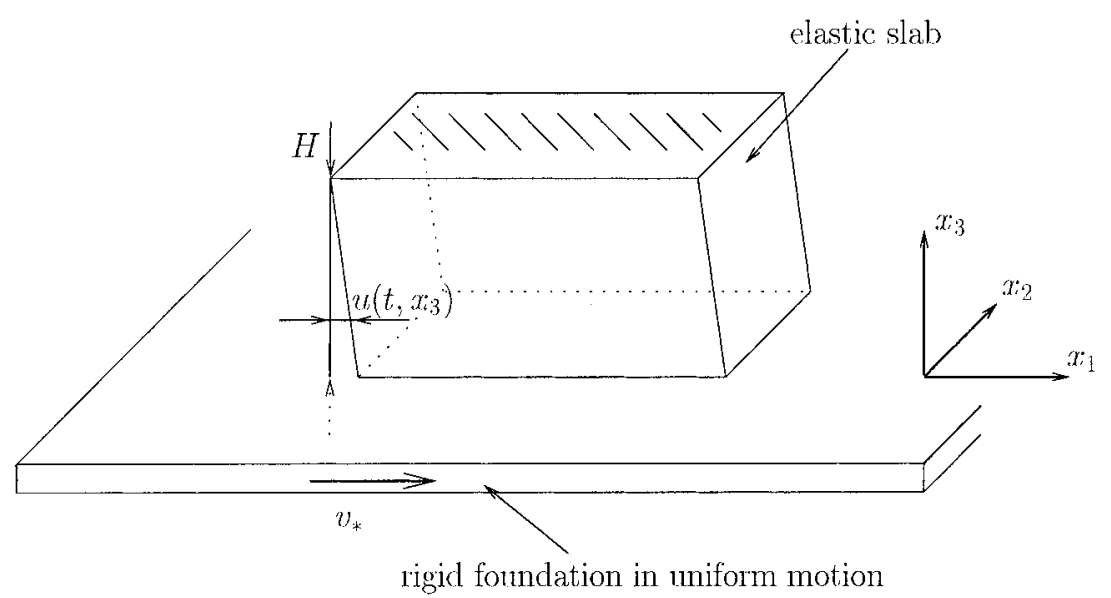

Fig. 1. Elastic slab in sliding with dry friction on a rigid foundation which is in uniform motion $v_{*}$ along the $x_{1}$ axis. 


$$
\begin{aligned}
& \forall t \in] 0, T] \\
& \text { if } \partial_{t} u_{T}(t, 0)=v_{*} \text { then }\left|G \partial_{x_{3}} u_{T}(t, 0)\right| \leqslant-(\lambda+2 G) \partial_{x_{3}} u_{3}(t, 0) \mu(0), \\
& \text { if } \partial_{t} u_{T}(t, 0) \neq v_{*} \text { then }\left\{\begin{array}{l}
\left|G \partial_{x_{3}} u_{T}(t, 0)\right|=-(\lambda+2 G) \partial_{x_{3}} u_{3}(t, 0) \mu\left(\left\|\partial_{t} u_{T}(t, 0)-v_{*}\right\|\right), \\
\exists \lambda \geqslant 0, \partial_{t} u_{T}(t, 0)-v_{*}=\lambda \partial_{x_{3}} u_{T}(t, 0),
\end{array}\right.
\end{aligned}
$$

where $\mu: \mathbb{R}^{+} \rightarrow \mathbb{R}^{+}$is the friction coefficient, and $v_{*}$ the constant planar velocity of the rigid foundation. It is easy to see that this friction condition is equivalent to the following inclusion:

$$
\begin{aligned}
& \forall t \in] 0, T], \\
& \quad G \partial_{x_{3}} u_{T}(t, 0) \in-(\lambda+2 G) \partial_{x_{3}} u_{3}(t, 0) \mu\left(\left\|\partial_{t} u_{T}(t, 0)-v_{*}\right\|\right) \operatorname{Dir}\left(\partial_{t} u_{T}(t, 0)-v_{*}\right),
\end{aligned}
$$

where the multi-valued map

$$
\operatorname{Dir}(v)=\left\{\frac{v}{\|v\|}\right\} \text { if } v \neq 0, \quad \operatorname{Dir}(0)=\bar{B}(0,1)
$$

is defined on $\mathbb{R}^{2}$, and is the sub-differential of the convex map $v \mapsto\|v\|$. The problem is completed by initial conditions

$$
\underline{u}\left(0, x_{3}\right)=\underline{u}^{0}\left(x_{3}\right), \quad \partial_{t} \underline{u}\left(0, x_{3}\right)=\underline{u}^{1}\left(x_{3}\right) \quad \forall x_{3} \in[0, H] .
$$

Finally, introducing the matrix

$$
\mathbf{C}=\left(\begin{array}{ccc}
c_{1} & 0 & 0 \\
0 & c_{1} & 0 \\
0 & 0 & c_{2}
\end{array}\right),
$$

and with the previous notations, the problem can be written as follows:

$$
\begin{aligned}
(P 1) \quad & \left.\left.\left.\partial_{t t}^{2} \underline{u}\left(t, x_{3}\right)=\mathbf{C} \partial_{x_{3} x_{3}}^{2} \underline{u}\left(t, x_{3}\right) \quad \text { in }\right] 0, T\right] \times\right] 0, H[, \\
& \underline{u}(t, H)=(0,0,-D) \quad \text { with } D>0 \quad \forall t \in] 0, T], \\
& \underline{u}\left(0, x_{3}\right)=\underline{u}^{0}\left(x_{3}\right), \quad \partial_{t} \underline{u}\left(0, x_{3}\right)=\underline{u}^{1}\left(x_{3}\right) \quad \forall x_{3} \in[0, H], \\
& \left.\left.(\lambda+2 G) \partial_{x_{3}} u_{3}(t, 0) \in-J_{N}\left(-u_{3}(t, 0)\right) \quad \forall t \in\right] 0, T\right], \\
& \left.\left.G \partial_{x_{3}} u_{T}(t, 0) \in-(\lambda+2 G) \partial_{x_{3}} u_{3}(t, 0) \mu\left(\left\|\partial_{t} u_{T}(t, 0)-v_{*}\right\|\right) \operatorname{Dir}\left(\partial_{t} u_{T}(t, 0)-v_{*}\right) \quad \forall t \in\right] 0, T\right],
\end{aligned}
$$

Due to the bad behavior of both the unilateral contact and the friction conditions, it cannot be expected to have any solution of the problem in a classical sense (i.e. twice continuously differentiable). It is easy to see that, most of the time, the velocity $\partial_{t} \underline{u}$ is not continuous (see [8]). One can also notice that it is possible to solve independently the problem in vertical displacement $u_{3}$.

Friction laws such as Coulomb law with a sliding velocity dependent coefficient have a long history. Rabinowicz already studies such dependencies in 1958 in [14]. Important experimental works on the measurement of this dependency has been made (see for instance [2,5,7,10,21]). Most of these experiments have been made on the steady state because transient states are extremely difficult to measure.

Very often, a decreasing coefficient of friction is used in order to modelize stick-slip phenomenon. The value of the coefficient for a sliding velocity equal to zero is called the static coefficient $\mu_{\mathrm{s}}$, and the asymptotic value for a huge sliding velocity is called the dynamic coefficient of friction $\mu_{\mathrm{d}}$.

\section{On the existence and uniqueness}

For convenience and from now on, we assume $T<\left(H / c_{2}\right)$. This is not strictly necessary, but the analysis will be greatly simplified, and this is not a real restriction, since all the following results can be extended for $T>\left(H / c_{2}\right)$ in studying the problem successively on intervals 


$$
\left[0,\left(\frac{H}{c_{2}}\right)\right], \quad\left[\frac{H}{c_{2}}, 2 \frac{H}{c_{2}}\right], \quad\left[2 \frac{H}{c_{2}}, 3 \frac{H}{c_{2}}\right], \ldots
$$

Let us introduce the quantities

$$
\begin{aligned}
& b_{+}\left(t, x_{3}\right)=\partial_{t} \underline{u}\left(t, x_{3}\right)+\mathbf{C}_{3} \underline{u}\left(t, x_{3}\right), \\
& b_{+}\left(t, x_{3}\right)=\partial_{t} \underline{u}\left(t, x_{3}\right)+\mathbf{C} \partial_{3} \underline{u}\left(t, x_{3}\right),
\end{aligned}
$$

which are propagated along the characteristic lines of Eq. (11), i.e. that for a convenient $\delta t$ and for $\varepsilon$ is + or - one has

$$
\begin{aligned}
& b_{1 \varepsilon}\left(t+\delta t, x_{3}\right)=b_{1 \varepsilon}\left(t, x_{3}+\varepsilon c_{1} \delta t\right), \\
& b_{2 \varepsilon}\left(t+\delta t, x_{3}\right)=b_{2 \varepsilon}\left(t, x_{3}+\varepsilon c_{1} \delta t\right), \\
& b_{3 \varepsilon}\left(t+\delta t, x_{3}\right)=b_{3 \varepsilon}\left(t, x_{3}+\varepsilon c_{2} \delta t\right),
\end{aligned}
$$

where $b_{1 \varepsilon}, b_{2 \varepsilon}$ and $b_{3 \varepsilon}$ are the components of $b_{\varepsilon}$. Since we assume $T<H / c_{2}$, and thanks to these equalities, if we set

$$
u_{N}(t)=-u_{3}(t, 0), \quad v_{\mathrm{s}}=\partial_{t} u_{T}(t, 0)-v_{*}, \quad b_{T \varepsilon}=\left(\begin{array}{c}
b_{1 \varepsilon} \\
b_{2 \varepsilon}
\end{array}\right),
$$

and if we denote by

$$
S(t)=-\frac{(\lambda+2 G)}{c_{2}}\left(b_{3+}(t, 0)+\frac{\mathrm{d}}{\mathrm{d} t} u_{N}(t)\right)
$$

the contact pressure which is always positive, then Problem (P1) becomes

$(P 2) \quad$ Find $u_{N}$ and $v_{\mathrm{s}}(t)$ such that:

$$
\begin{aligned}
& \frac{\mathrm{d}}{\mathrm{d} t} u_{N}(t) \in-b_{3+}(t, 0)-\frac{c_{2}}{\lambda+2 G} J_{N}\left(u_{N}(t)\right), \\
& u_{N}(0)=u_{N 0}=u_{3}^{0}(0,0), \\
& \frac{G}{c_{1}}\left(b_{T+}-v_{*}\right) \in \frac{G}{c_{1}} v_{\mathrm{s}}(t)+S(t) \mu\left(\left\|v_{\mathrm{s}}(t)\right\|\right) \operatorname{Dir}\left(v_{\mathrm{s}}(t)\right) .
\end{aligned}
$$

One can notice that those two equations are not of the same kind. Eqs. (23) and (24) form a first-order differential inclusion Cauchy problem in the variable $u_{N}$, and Eq. (25) is a scalar inclusion in variable $v_{\mathrm{s}}$. Problem (23), (24) is still independent of Eq. (25) and the following result holds:

Proposition 1. If $b_{3+}(., 0) \in L^{1}(0, T)$ and if the initial condition satisfies $u_{N 0} \leqslant 0$, the following Cauchy problem

$$
\left\{\begin{array}{l}
\frac{\mathrm{d}}{\mathrm{d} t} u_{N}(t) \in-b_{3+}(t, 0)-\frac{c_{2}}{\lambda+2 G} J_{N}\left(u_{N}(t)\right), \quad \text { a.e. in }[0, T], \\
u_{N}(0)=u_{N 0},
\end{array}\right.
$$

has a unique absolutely continuous solution, and this solution satisfies $u_{N}(t) \leqslant 0$ on $[0, T]$.

The proof of this result comes directly from the result of Deimling [4] on differential inclusions, and the fact that the multi-valued map

$$
(t, x) \mapsto-b_{3+}(t, 0)-\frac{c_{2}}{\lambda+2 G} J_{N}(x),
$$

has convex values, is measurable, upper semi-continuous and one-sided Lipschitz.

Unfortunately, inclusion (25) has generally not a unique solution. We can only prove the following result: 
Proposition 2. If the friction coefficient $\mu$ is a Lipschitz continuous and bounded positive function of the sliding velocity $v_{\mathrm{s}}$, then Inclusion (25) has at least one solution. Moreover, if the friction coefficient $\mu$ is an increasing function of $v_{\mathrm{s}}$ this solution is unique.

The proof of this result is a simple study of the shape of the right-hand side of Inclusion (25).

In fact, when the friction coefficient has at least one decreasing branch, it is always possible to exhibit a case where there is more than one solution to Inclusion (25). See [8,19] for such examples. Moreover, this is the interesting case, since it seems to be necessary to use a coefficient with a decreasing branch to modelize well stick-slip phenomena.

\section{Mass perturbation and perfect delay criterion}

One of the major problems is thus the non-uniqueness of the solution of Problem (P1). In fact, certain considerations of mechanical order (see [19]) allow to say that most of the solutions of Inclusion (25) are not acceptable. Authors like $[8,10]$ proposed a criterion in order to choose the convenient solution of the problem. This criterion is a dynamic extension of the perfect delay criterion which comes from the catastrophe theory and which can be formulated as follows:

the system only jump when it has no other choice.

This criterion is not sufficiently accurate to always select a unique solution (it does in experience of pure loading or pure unloading, see [8]).

Another approach to this problem is to introduce a viscous term in the elastic law and to make this term to vanish. In this framework, it is possible to prove that the visco-elastic version of the problem has a unique solution. But at this moment there is no theoretical results of convergence when the viscous term vanishes.

The approach we introduced in a work [19], is to consider a mass perturbation of the friction condition. The classical friction condition

$$
G \partial_{x_{3}} u_{T}(t, 0) \in S(t) \mu\left(\left\|\partial_{t} u_{T}(t, 0)-v_{*}\right\|\right) \operatorname{Dir}\left(\partial_{t} u_{T}(t, 0)-v_{*}\right),
$$

is replaced by the following condition

$$
\varepsilon \partial_{t t}^{2} u_{T}+G \partial_{x_{3}} u_{T}(t, 0) \in S(t) \mu\left(\left\|\partial_{t} u_{T}(t, 0)-v_{*}\right\|\right) \operatorname{Dir}\left(\partial_{t} u_{T}(t, 0)-v_{*}\right),
$$

where $\varepsilon$ is the perturbation parameter. The additional term $\varepsilon \partial_{t t}^{2} u_{T}$ can be assimilated as a surface mass on the contact boundary. This kind of perturbations, similarly to existing friction laws as Dieterich-Ruina laws (see [20]), make a differential operator to appear on the contact boundary. Further development of such boundary perturbations in upper dimensions are presented in [13].

With this mass perturbation, the Problem (P2) becomes

(P3) Find $u_{N}$ and $v_{s}(t)$ suchthat:

$$
\begin{aligned}
& \frac{\mathrm{d}}{\mathrm{d} t} u_{N}(t) \in-b_{3+}(t, 0)-\frac{c_{2}}{\lambda+2 G} J_{N}\left(u_{N}(t)\right), \\
& u_{N}(0)=u_{N}^{0}=u_{3}^{0}(0,0), \\
& \varepsilon \frac{\mathrm{d}}{\mathrm{d} t} v_{\mathrm{s}}(t) \in \frac{G}{c_{1}}\left(b_{T+}+v_{\mathrm{s}}(t)-v_{*}\right)+S(t) \mu\left(\left\|v_{\mathrm{s}}(t)\right\|\right) \operatorname{Dir}\left(v_{\mathrm{s}}(t)\right), \\
& v_{\mathrm{s}}(0)=v_{\mathrm{s}}^{0}=u_{T}^{1}(0,0) .
\end{aligned}
$$

The first result is that Problem (P3) has a unique solution. 
Proposition 3. If $b_{T^{+}} \in\left(L^{1}(0, T)\right)^{2}$ and if the contact pressure $S$ is a positive function and belongs to $L^{1}(0, T)$ (this is the case if $S$ comes from the solution of (31), (32) and if conditions of Proposition (1) hold), then the following Cauchy problem:

$$
\left\{\begin{array}{l}
\varepsilon \frac{\mathrm{d}}{\mathrm{d} t} v_{\mathrm{s}}(t) \in \frac{G}{c_{1}}\left(b_{T^{+}}+v_{\mathrm{s}}(t)-v_{*}\right)+S(t) \mu\left(\left\|v_{\mathrm{s}}(t)\right\|\right) \operatorname{Dir}\left(v_{\mathrm{s}}(t)\right), \\
v_{\mathrm{s}}(0)=v_{\mathrm{s}}^{0}
\end{array}\right.
$$

has a unique absolutely continuous solution.

Similarly as for Proposition 1, this result comes from the fact that the multi-valued map

$$
(t, v) \mapsto \frac{G}{c_{1}}\left(b_{T+}+v-v_{*}\right)+S(t) \mu(\|v\|) \operatorname{Dir}(v),
$$

has convex values, is upper semi-continuous and one-sided Lipschitz.

In a more restrictive framework, in [19], we proved that when $\varepsilon$ goes to zero the solution of the perturbed problem converges toward a particular solution of the initial problem. The connection between this and the perfect delay criterion is that if this criterion selects a unique solution, then this is also the limit of the solutions of the perturbed problems.

\section{Regularized unilateral contact condition}

For the convergence proof of Section 6 , we need the solution to have a certain regularity. The regularity of $u_{N}$ is not sufficient. One of the most usual way to regularize the unilateral contact condition is to consider a continuous approximation of the map $J_{N}$. It is the case, for example, for the well-known normal compliance law developed by Oden and Martins in [12]. Here we consider the following simple piecewise linear approximation $J_{N}^{\eta}$ of $J_{N}$ :

$$
J_{N}^{\eta}(x)= \begin{cases}0 & \text { if } x<0 \\ \frac{x}{\eta} & \text { if } x \geqslant 0\end{cases}
$$

The solutions of the differential equation

$$
\frac{\mathrm{d}}{\mathrm{d} t} u_{N}(t)=-b_{3+}(t, 0)-\frac{c_{2}}{\lambda+2 G} J_{N}^{\eta}\left(u_{N}(t)\right)
$$

are such that the time derivative of $u_{N}$ is Lipschitz continuous since $b_{3+}(t, 0)$ is. This regularization corresponds to a certain interface elasticity.

\section{Numerical analysis}

In this section we present a numerical scheme for the perturbed problem. Once again, the results will be established for $T<H / c_{2}$ even though they can be easily extended for $T>H / c_{2}$ and of course even though the numerical scheme has not himself such a restriction. The scheme is a coupling of a Lax-Wendroff scheme for the interior of the slab and of implicit Euler schemes for the equations on the boundary. The implicit Euler scheme is chosen for the simplicity of the proof. More accurate schemes can be used (in [18] we use a theta method, with a parameter $1 / 2$, which is also an two points Adams-Moulton scheme, and gives a better numerical approximation). The convergence is obtained for the regularized unilateral contact condition. This is an open problem to know if it should be possible to work with the normal contact condition. This analysis can be viewed as a first step to improve numerical analysis results for purely elastic friction problems with a complex friction law. 


\subsection{Description of the scheme}

The slab is discretized with a constant step $\Delta z=H / N_{z}$ and a constant time step $\Delta t=T / N_{t}$. The principal variables are $\underline{u}_{, t}=\partial_{t} \underline{u}$ and $\underline{u}_{, 3}=\partial_{x_{3}} \underline{u}$. Let us denote by

$$
\underline{u}^{i, n}=\left(\begin{array}{c}
u_{1}^{i, n} \\
u_{2}^{i, n} \\
u_{3}^{i, n}
\end{array}\right), \quad \underline{u}_{, t}^{i, n}=\left(\begin{array}{c}
u_{1, t}^{i, n} \\
u_{2, t}^{i, n} \\
u_{3, t}^{i, n}
\end{array}\right), \quad \text { and } \quad \underline{u}_{, 3}^{i, n}=\left(\begin{array}{c}
u_{1,3}^{i, n} \\
u_{2,3}^{i, n} \\
u_{3,3}^{i, n}
\end{array}\right) \text {, }
$$

the approximated values of, respectively $\underline{u}_{,} \underline{u}_{t}$ and $\underline{u}_{3}$ at the time $t^{n}=n \Delta t$ and at $x_{3}^{i}=i \Delta z$. We also use the following notations for the tangential part:

$$
\underline{u}_{T}^{0, n}=\left(\begin{array}{c}
u_{1}^{0, n} \\
u_{2}^{0, n}
\end{array}\right), \quad \underline{u}_{T, t}^{0, n}=\left(\begin{array}{c}
u_{1, t}^{0, n} \\
u_{2, t}^{0, n}
\end{array}\right) \quad \text { and } \quad \underline{u}_{T, 3}^{0, n}=\left(\begin{array}{c}
u_{1,3}^{0, n} \\
u_{2,3}^{0, n}
\end{array}\right) .
$$

With the matrix

$$
\mathbf{D}=\frac{\Delta t}{\Delta z} C=\left(\begin{array}{cccc}
c_{1} & \frac{\Delta t}{\Delta z} & 0 & 0 \\
0 & c_{1} & \frac{\Delta t}{\Delta z} & 0 \\
0 & 0 & c_{2} & \frac{\Delta t}{\Delta z}
\end{array}\right)=\left(\begin{array}{ccc}
d_{1} & 0 & 0 \\
0 & d_{1} & 0 \\
0 & 0 & d_{2}
\end{array}\right)
$$

The Lax-Wendroff scheme on the interior of the slab, for $1 \leqslant i \leqslant N_{z}-1$, reads

$$
\begin{aligned}
& \underline{u}_{, t}^{i, n+1}=\underline{u}_{, t}^{i, n}+\frac{\mathbf{D}}{2}\left(\underline{u}_{, t}^{i-1, n}-2 \underline{u}_{, t}^{i, n}+\underline{u}_{, t}^{i+1, n}\right)+\frac{\mathbf{D C}}{2}\left(\underline{u}_{, 3}^{i+1, n}-\underline{u}_{, 3}^{i-1, n}\right), \\
& \underline{u}_{, 3}^{i, n+1}=\underline{u}_{, 3}^{i, n}+\frac{\mathbf{D}}{2}\left(\underline{u}_{3}^{i-1, n}-2 \underline{u}_{, 3}^{i, n}+\underline{u}_{, 3}^{i+1, n}\right)+\frac{\mathbf{D C}}{2}\left(\underline{u}_{, t}^{i+1, n}-\underline{u}_{, t}^{i-1, n}\right) .
\end{aligned}
$$

With

$$
\underline{b}_{+}^{i, n}=\underline{u}_{, t}^{i, n}+\mathbf{C} \underline{u}_{, 3}^{i, n} \quad \text { and } \quad \underline{b}_{-}^{i, n}=\underline{u}_{, t}^{i, n}-\mathbf{C} \underline{u}_{, 3}^{i, n},
$$

this scheme reads also

$$
\begin{array}{ll}
\underline{b}_{+}^{i, n+1}=\underline{b}_{+}^{i, n}+\mathbf{D}\left(\underline{b}_{+}^{i+1, n}-\underline{b}_{+}^{i, n}\right), & 0 \leqslant i \leqslant N_{z}-1, \\
\underline{b}_{-}^{i, n+1}=\underline{b}_{-}^{i, n}+\mathbf{D}\left(\underline{b}_{-}^{i-1, n}-\underline{b}_{-}^{i, n}\right), & 1 \leqslant i \leqslant N_{z},
\end{array}
$$

The approximation of the Dirichlet condition on $z=H$ is

$$
\begin{aligned}
& \underline{u}_{, 3}^{N_{z}, n+1}=\underline{u}_{, 3}^{N_{z}, n}+\mathbf{D}\left(\underline{u}_{, 3}^{N_{z}-1, n}-\underline{u}_{3}^{N_{z}, n}\right)-\mathbf{D} \mathbf{C}^{-1} \underline{u}_{, t}^{N_{z}-1, n}, \\
& \underline{u}_{, t}^{N_{z}, n+1}=0 .
\end{aligned}
$$

or

$$
\underline{b}_{+}^{N_{z}, n+1}=-\underline{b}_{-}^{N_{z}, n+1} .
$$

The unilateral contact condition, which corresponds to the differential inclusion

$$
\frac{\mathrm{d}}{\mathrm{d} t} u_{3}(t, 0) \in b_{3+}(t, 0)+J_{N}\left(-u_{3}(t, 0)\right),
$$

for the nonregularized case or to the differential equation

$$
\frac{\mathrm{d}}{\mathrm{d} t} u_{3}(t, 0)=b_{3+}(t, 0)+\frac{c_{2}}{\lambda+2 G} J_{N}^{\eta}\left(-u_{3}(t, 0)\right)
$$

for the regularized case, is approximated with an implicit Euler scheme. This gives for the non regularized case 


$$
u_{3}^{0, n+1}=\left(u_{3}^{0, n}+\Delta t b_{3+}^{0, n+1}\right)_{+},
$$

where $(x)_{+}=\max (0, x)$ is the positive part of $x$. The value of $u_{3, t}^{0, n+1}$ is approximated by

$$
u_{3, t}^{0, n+1}= \begin{cases}b_{3+}^{0, n+1} & \text { if } u_{3}^{0, n+1} \neq 0 \\ 0 & \text { otherwise }\end{cases}
$$

For the regularized case, the implicit Euler scheme gives

$$
\begin{aligned}
& u_{3}^{0, n+1}=\left(u_{3}^{0, n}+\Delta t b_{3+}^{0, n+1}\right)_{+}+\frac{1}{1+\frac{\Delta t}{\tilde{\eta}}}\left(u_{3}^{0, n}+\Delta t b_{3+}^{0, n+1}\right)_{-}, \\
& u_{3, t}^{0, n+1}=b_{3+}^{0, n+1}+J_{N}^{\tilde{\eta}}\left(-u_{3}^{0, n+1}\right),
\end{aligned}
$$

where $\tilde{\eta}=\frac{\lambda+2 G}{c_{2}} \eta$.

The contact pressure is thus given by

$$
S^{n+1}=-\frac{\lambda+2 G}{c_{2}}\left(b_{3+}^{0, n+1}-u_{3, t}^{0, n+1}\right) .
$$

Those schemes are such that $S^{n+1} \geqslant 0$. Moreover, for the nonregularized case, one has

$$
u_{3}^{0, n+1} \geqslant 0, \quad S^{n+1} u_{3}^{0, n+1}=0,
$$

similarly as the continuous case.

Now, concerning the perturbed friction condition, we have to approximate the following differential inclusion:

$$
\varepsilon \frac{\mathrm{d}}{\mathrm{d} t} \underline{u}_{T, t} \in \frac{G}{c_{1}}\left(b_{T+}(t)-\underline{u}_{T, t}\right)-S(t) \mu\left(\left\|\underline{u}_{T, t}-\underline{V}_{e}(t)\right\|\right) \operatorname{Dir}\left(\underline{u}_{T, t}-\underline{V}_{e}(t)\right),
$$

The implicit Euler scheme reads

$$
\underline{u}_{T, t}^{0, n+1} \in \underline{u}_{T, t}^{0, n}+\frac{\Delta t}{\varepsilon}\left(\frac{G}{c_{1}}\left(b_{T+}^{0, n+1}-\underline{u}_{T, t}^{0, n+1}\right)-S^{n+1} \mu\left(\left\|\underline{u}_{T, t}^{0, n+1}-\underline{V}_{e}\left(t^{n+1}\right)\right\|\right) \operatorname{Dir}\left(\underline{u}_{T, t}^{0, n+1}-\underline{V}_{e}\left(t^{n+1}\right)\right)\right),
$$

\subsection{Properties of the Lax-Wendroff scheme}

It is well known that the Lax-Wendroff scheme is not stable if the Courant-Friedrichs-Levi condition $d_{2}=\frac{\Delta t}{\Delta z} c_{2} \leqslant 1$ does not hold. Thus we assume that the matrix $\mathbf{D}$ has all its coefficients between 0 and 1 .

The Lax-Wendroff scheme has important properties concerning the $L^{\infty}$ stability. If we consider the following quantities:

$$
\begin{aligned}
B_{\max }^{n} & =\max _{0 \leqslant i \leqslant N_{z}}\left(\left\|\underline{b}_{+}^{i, n}\right\|,\left\|\underline{b}_{-}^{i, n}\right\|\right), \\
d B_{\max }^{n} & =\max _{0 \leqslant i \leqslant N_{z}-1}\left(\left\|\underline{b}_{+}^{i+1, n}-\underline{b}_{+}^{i, n}\right\|,\left\|\underline{b}_{-}^{i+1, n}-\underline{b}_{-}^{i, n}\right\|\right),
\end{aligned}
$$

then from Eqs. (42) and (43) it comes

$$
\begin{aligned}
\left\|\underline{b}_{+}^{i, n+1}\right\| & =\left\|\underline{b}_{+}^{i, n}+\mathbf{D}\left(\underline{b}_{+}^{i+1, n}-\underline{b}_{+}^{i, n}\right)\right\| \leqslant B_{\max }^{n}, & & 0 \leqslant i \leqslant N_{z}-1, \\
\left\|\underline{b}_{-}^{i, n+1}\right\| & =\left\|\underline{b}_{-}^{i, n}+\mathbf{D}\left(\underline{b}_{-}^{i-1, n}-\underline{b}_{-}^{i, n}\right)\right\| \leqslant B_{\max }^{n}, & & 1 \leqslant i \leqslant N_{z},
\end{aligned}
$$

and

$$
\begin{aligned}
\left\|\underline{b}_{+}^{i, n+1}-\underline{b}_{+}^{i+1, n+1}\right\| & =\left\|\underline{b}_{+}^{i, n}-\underline{b}_{+}^{i+1, n}+\mathbf{D}\left(\underline{b}_{+}^{i+1, n}-\underline{b}_{+}^{i+2, n}-\underline{b}_{+}^{i, n}+\underline{b}_{+}^{i+1, n}\right)\right\| \\
& =d B_{\max }^{n}, \quad 0 \leqslant i \leqslant N_{z}-2, \\
\left\|\underline{b}_{-}^{i, n+1}-\underline{b}_{-}^{i-1, n+1}\right\| & \leqslant\left\|\underline{b}_{-}^{i, n}-\underline{b}_{-}^{i-1, n}+\mathbf{D}\left(\underline{b}_{-}^{i-1, n}-\underline{b}_{-}^{i-2, n}-\underline{b}_{-}^{i, n}+\underline{b}_{-}^{i-1, n}\right)\right\| \\
& \leqslant d B_{\max }^{n}, \quad 2 \leqslant i \leqslant N_{z} .
\end{aligned}
$$


That is to say that the Lax-Wendroff scheme on the interior of the slab makes $B_{\max }^{n}$ and $d B_{\max }^{n}$ only to decrease. It is not difficult to see that the Dirichlet condition do the same

$$
\begin{aligned}
\left\|\underline{b}_{+}^{N_{z}, n+1}\right\| & =\left\|\underline{b}_{-}^{N_{z}, n+1}\right\| \leqslant B_{\max }^{n}, \\
\left\|\underline{b}_{+}^{N_{z}-1, n+1}-\underline{b}_{+}^{N_{z}, n+1}\right\| & =\left\|\underline{b}_{+}^{N_{z}-1, n}+\mathbf{D}\left(\underline{b}_{+}^{N_{z}, n}-\underline{b}_{+}^{N_{z}-1, n}\right)-\underline{b}_{+}^{N_{z}, n}+\mathbf{D}\left(\underline{b}_{-}^{N_{z}-1, n}-\underline{b}_{-}^{N_{z}, n}\right)\right\|, \\
& \leqslant d B_{\max }^{n} .
\end{aligned}
$$

Thus we can conclude

$$
\begin{aligned}
& B_{\max }^{n+1} \leqslant \max \left(B_{\max }^{n},\left\|\underline{b}_{-}^{0, n+1}\right\|\right), \\
& d B_{\max }^{n+1} \leqslant \max \left(d B_{\max }^{n},\left\|\underline{b}_{-}^{1, n+1}-\underline{b}_{-}^{0, n+1}\right\|\right) .
\end{aligned}
$$

Moreover, for $n \leqslant N_{z}$, the following estimation holds:

$$
\begin{aligned}
& \left\|\underline{b}_{+}^{0, n}\right\| \leqslant B_{\max }^{0}, \\
& \left\|\underline{b}_{+}^{1, n}-\underline{b}_{+}^{0, n}\right\| \leqslant d B_{\max }^{0} .
\end{aligned}
$$

The reason is that if one develops $\underline{b}_{+}^{0, n}$, one has

$$
\begin{aligned}
\underline{b}_{+}^{0, n} & =(1-\mathbf{D}) \underline{b}_{+}^{0, n-1}+\mathbf{D} \underline{b}_{+}^{1, n-1}, \\
& =(1-\mathbf{D})^{2} \underline{b}_{+}^{0, n-2}+2(1-\mathbf{D}) \mathbf{D} \underline{b}_{+}^{1, n-2}+\mathbf{D}^{2} \underline{b}_{+}^{2, n-2}, \\
& =\ldots \\
& =\sum_{i=0}^{j}\left(\begin{array}{c}
j \\
i
\end{array}\right) \mathbf{D}^{i}(1-\mathbf{D})^{j-i} \underline{b}_{+}^{i, n-j}, \quad \text { for } 0 \leqslant j \leqslant \min \left(n, N_{z}\right),
\end{aligned}
$$

where the $\left(\begin{array}{c}j \\ i\end{array}\right)$ are the combinations of $i$ elements among $j$. Since $\sum_{i=0}^{j}\left(\begin{array}{l}j \\ i\end{array}\right) \mathbf{D}^{i}(1-\mathbf{D})^{j-i}=$ $(1-\mathbf{D}+\mathbf{D})^{j}=1$ the result hold. The same computation can be made with $\left\|\underline{b}_{+}^{1, n}-\underline{b}_{+}^{0, n}\right\|$.

\subsection{Stability of the schemes}

We first deal with the $u_{3}$ component of the displacement, i.e. the component submitted to the unilateral contact condition. We consider $B_{3 \max }^{n}$ and $d B_{3 \max }^{n}$ :

$$
\begin{aligned}
B_{3 \max }^{n} & =\max _{0 \leqslant i \leqslant N_{z}}\left(\left|b_{3+}^{i, n}\right|,\left|b_{3-}^{i, n}\right|\right), \\
d B_{3 \max }^{n} & =\max _{0 \leqslant i \leqslant N_{-}-1}\left(\left|b_{3+}^{i+1, n}-b_{3+}^{i, n}\right|,\left|b_{3-}^{i+1, n}-b_{3-}^{i, n}\right|\right),
\end{aligned}
$$

We just saw that

$$
\begin{aligned}
& B_{3 \max }^{n+1} \leqslant \max \left(B_{3 \max }^{n},\left|b_{3-}^{0, n+1}\right|\right), \\
& d B_{3 \max }^{n+1} \leqslant \max \left(d B_{3 \text { max }}^{n},\left|b_{3-}^{1, n+1}-b_{3-}^{0, n+1}\right|\right) .
\end{aligned}
$$

Proposition 4. If the CFL condition $d_{2} \leqslant 1$ holds, schemes (39)-(46), (47), (48) has the following properties:

$$
\begin{aligned}
& B_{3 \max }^{n} \leqslant B_{3 \max }^{0}, \\
& \left|u_{3}^{0, n}\right| \leqslant\left|u_{3}^{0,0}\right|+T B_{3 \max }^{0} .
\end{aligned}
$$

Proof. We have

$$
b_{3-}^{0, n+1}= \begin{cases}-b_{3+}^{0, n+1} & \text { if } u_{3, t}^{0, n+1}=0, \\ b_{3+}^{0, n+1} & \text { if } u_{3, t}^{0, n+1}=b_{3+}^{0, n+1}\end{cases}
$$


Thus $\left|b_{3-}^{0, n+1}\right|=\left|b_{3+}^{0, n+1}\right|$ and

$$
B_{3 \max }^{n+1} \leqslant B_{3 \max }^{n} \leqslant \cdots \leqslant B_{3 \max }^{0} .
$$

For $u_{3}^{0, n}$ now we have

$$
\left|u_{3}^{0, n+1}-u_{3}^{0, n}\right| \leqslant \Delta t\left|b_{3+}^{0, n+1}\right|
$$

from the scheme, and thus

$$
\left|u_{3}^{0, n}\right| \leqslant T B_{3 \max }^{0} .
$$

Proposition 5. If exists $d_{\min }>0$ such that $d_{\min } \leqslant d_{2} \leqslant 1$ then schemes (39)-(46), (49), (50) have the following properties: Exist $C_{1}, C_{2}, C_{3}$ and $C_{4}$ four positive constants which only depend on initial data such that

$$
\begin{aligned}
B_{3 \max }^{n} & \leqslant C_{1}, \\
\left|u_{3}^{0, n}\right| & \leqslant C_{2}, \\
d B_{3 \max }^{n} & \leqslant C_{3} d B_{3 \max }^{0}+\Delta t C_{4} .
\end{aligned}
$$

Proof. We saw that

$$
\left|b_{3+}^{0, n}\right| \leqslant B_{3 \max }^{0}, \quad\left|b_{3+}^{1, n}-b_{3+}^{0, n}\right| \leqslant d B_{3 \max }^{0},
$$

and it follows from scheme (49), (50) that

$$
\begin{aligned}
\left|u_{3}^{0, n+1}\right| & \leqslant\left|\Delta t b_{3+}^{0, n+1}+u_{3}^{0, n}\right|, \\
& \leqslant\left|u_{3}^{0, n}\right|+\Delta t B_{3 \max }^{0},
\end{aligned}
$$

thus, since we assume $n<N_{z}$ :

$$
\begin{aligned}
\left|u_{3}^{0, n}\right| & \leqslant\left|u_{3}^{0,0}\right|+N_{z} \Delta t B_{3 \max }^{0}, \\
& \leqslant\left|u_{3}^{0,0}\right|+\frac{H}{c_{2}} B_{3 \max }^{0} .
\end{aligned}
$$

Moreover, we can also deduce from schemes (49), (50) that

$$
\begin{aligned}
\left|u_{3}^{0, n+1}-u_{3}^{0, n}\right| & \leqslant \Delta t\left(\left|b_{3+}^{0, n+1}\right|+\frac{\left|u_{3}^{0, n+1}\right|}{\tilde{\eta}}\right) \\
& \leqslant \Delta t B_{3 \max }^{0}\left(1+2 N_{z} \Delta t+\left|u_{3}^{0,0}\right|\right) .
\end{aligned}
$$

and because

$$
b_{3-}^{0, n+1}=b_{3+}^{0, n+1}+2 c_{2} u_{3,3}^{0, n+1},
$$

and

$$
u_{3,3}^{0, n+1}=\left\{\begin{array}{l}
0 \quad \text { if } u_{3}^{0, n+1} \geqslant 0 \\
\frac{u_{3}^{0, n+1}}{c_{2} \tilde{\eta}} \quad \text { if } u_{3}^{0, n+1}<0
\end{array}\right.
$$

we have

$$
\begin{aligned}
\left|b_{3-}^{0, n+1}\right| & \leqslant\left|b_{3+}^{0, n+1}\right|+\frac{2\left|u_{3}^{0, n+1}\right|}{\tilde{\eta}} \\
& \leqslant B_{3 \max }^{0}\left(1+2 \frac{H}{c_{2}}\right)+2\left|u_{3}^{0,0}\right| .
\end{aligned}
$$


That is to say

$$
B_{3 \max }^{n} \leqslant B_{3 \max }^{0}\left(1+2 \frac{H}{c_{2}}\right)+2\left|u_{3}^{0,0}\right| .
$$

Now if we compute

$$
\begin{aligned}
\left|b_{3-}^{1, n+1}-b_{3-}^{0, n+1}\right| & =\left|b_{3-}^{1, n}+D\left(b_{3-}^{0, n}-b_{3-}^{1, n}\right)-b_{3-}^{0, n+1}\right| \\
& \leqslant\left(1-d_{2}\right)\left|b_{3-}^{1, n}-b_{3-}^{0, n}\right|+2\left|c_{2} u_{3,3}^{0, n}-c_{2} u_{3,3}^{0, n+1}\right|+\left|b_{3+}^{0, n+1}-b_{3+}^{0, n}\right|, \\
& \leqslant\left(1-d_{2}\right) d B_{3 \max }^{n}+d_{2} d B_{3 \max }^{0}+2 \frac{\Delta t}{\tilde{\eta}} B_{3 \max }^{0}\left(1+N_{z} \Delta t+\left|u_{3}^{0,0}\right|\right),
\end{aligned}
$$

it comes

$$
d B_{3 \max }^{n+1} \leqslant\left(1-d_{2}\right) d B_{3 \max }^{n}+d_{2} d B_{3 \max }^{0}+2 \frac{\Delta t}{\tilde{\eta}} B_{3 \max }^{0}\left(1+2 N_{z} \Delta t+\left|u_{3}^{0,0}\right|\right),
$$

and by induction

$$
\begin{aligned}
d B_{3 \max }^{n} & \leqslant\left(1-d_{2}\right)^{n} d B_{3 \max }^{0}+\left(d_{2} d B_{3 \max }^{0}+2 \frac{\Delta t}{\tilde{\eta}} B_{3 \max }^{0}\left(1+N_{z} \Delta t\right)\right) \sum_{i=0}^{n-1}\left(1-d_{2}\right)^{i} \\
& \leqslant\left(1-d_{2}\right)^{n} d B_{3 \max }^{0}+\frac{1}{d_{2}}\left(d_{2} d B_{3 \max }^{0}+2 \frac{\Delta t}{\tilde{\eta}} B_{3 \max }^{0}\left(1+N_{z} \Delta t+\left|u_{3}^{0,0}\right|\right)\right) \\
& \leqslant\left(1+\left(1-d_{2}\right)^{n}\right) d B_{3 \max }^{0}+\Delta t \frac{2}{\tilde{\eta} d_{2}} B_{3 \max }^{0}\left(1+\frac{H}{c_{2}}\right) \\
& \leqslant 2 d B_{3 \max }^{0}+\Delta t \frac{2}{\tilde{\eta} d_{\min }} B_{3 \max }^{0}\left(1+\frac{H}{c_{2}}+\left|u_{3}^{0,0}\right|\right)
\end{aligned}
$$

Now, for the complete scheme, including the friction condition, we consider $B_{T \max }^{n}$ and $d B_{T \max }^{n}$ :

$$
\begin{aligned}
B_{T \max }^{n} & =\max _{0 \leqslant i \leqslant N_{z}}\left(\left\|\underline{b}_{T+}^{i, n}\right\|,\left\|\underline{b}_{T-}^{i, n}\right\|\right), \\
d B_{T \max }^{n} & =\max _{0 \leqslant i \leqslant N_{z}-1}\left(\left\|\underline{b}_{T+}^{i+1, n}-\underline{b}_{T+}^{i, n}\right\|,\left\|\underline{b}_{T-}^{i+1, n}-\underline{b}_{T-}^{i, n}\right\|\right),
\end{aligned}
$$

where $\underline{b}_{T+}^{i, n}$ and $\underline{b}_{T-}^{i, n}$ are the horizontal components of $\underline{b}_{T}^{i, n}$ and $\underline{b}_{-}^{i, n}$.

Proposition 6. If exists $d_{\min }>0$ such that $d_{\min } \leqslant d_{2} \leqslant 1$ then schemes (39)-(46), (51), (52) combined with either schemes (47), (48) or (49), (50) has the following properties: there exist four positive constant $C_{1}, C_{2}, C_{3}$ and $C_{4}$ which only depend on the initial condition such that

$$
\begin{aligned}
B_{T \max }^{n} & \leqslant C_{1}, \\
\left\|u_{3}^{0, n}\right\| & \leqslant C_{2}, \\
d B_{T \text { max }}^{n} & \leqslant C_{3} d B_{T \text { max }}^{0}+\Delta t C_{4} .
\end{aligned}
$$

Proof. From (52), it comes

$$
\underline{u}_{T, t}^{0, n+1} \in \underline{u}_{T, t}^{0, n}+\frac{\Delta t}{\varepsilon}\left(\frac{G}{c_{1}}\left(b_{T+}^{0, n+1}-\underline{u}_{T, t}^{0, n+1}\right)-S^{n+1} \mu\left(\left\|\underline{u}_{T, t}^{0, n+1}-\underline{V}_{e}\left(t^{n+1}\right)\right\|\right) \underline{\operatorname{Dir}}\left(\underline{u}_{T, t}^{0, n+1}-\underline{V}_{e}\left(t^{n+1}\right)\right)\right),
$$

i.e.

$$
\underline{u}_{T, t}^{0, n+1} \in \underline{u}_{T, t}^{0, n}+\Delta t F\left(t^{n+1}, \underline{u}_{T, t}^{0, n+1}\right),
$$


with a multi-valued map $F$ which has a bounded growth

$$
\left\|F\left(t^{n}, x\right)\right\| \leqslant c\left(t^{n}\right)(1+\|x\|),
$$

with $c\left(t^{n}\right)$ defined by

$$
c\left(t^{n}\right)=\frac{1}{\varepsilon} \max \left(\frac{G}{c_{1}}, 1\right)\left(\left\|\underline{b}_{T+}^{0, n}\right\|+\left\|S^{n}\right\|\|\mu\|_{\infty}\right) .
$$

From the previous propositions, $\left\|S^{n}\right\|$ is bounded independently of $n$. Moreover, since we assume $n<N_{z}$, $\left\|\underline{b}_{T+}^{0, n}\right\| \leqslant B_{T \max }^{0}$ the quantity $c\left(t^{n}\right)$ is bounded independently of $n$ and $\Delta t$ for $t<\frac{H}{c_{2}} d_{\min }$. Setting

$$
c_{\max }=\max _{0 \leqslant i<2 N_{z}} c\left(t^{i}\right),
$$

and from (62) we deduce that

$$
\left\|\underline{u}_{T, t}^{0, n+1}\right\| \leqslant\left\|\underline{u}_{T, t}^{0, n}\right\|+\Delta t c_{\max }\left(1+\left\|\underline{u}_{T, t}^{0, n+1}\right\|\right) .
$$

We assume $\Delta t<\frac{1}{2 c_{\max }}$, thus

$$
\begin{aligned}
\left\|\underline{u}_{T, t}^{0, n+1}\right\| & \leqslant \frac{1}{1-\Delta t c_{\max }}\left(\left\|\underline{u}_{T, t}^{0, n}\right\|+c_{\max } \Delta t\right) \\
& \leqslant\left(1+2 c_{\max } \Delta t\right)\left(\left\|\underline{u}_{T, t}^{0, n}\right\|+c_{\max } \Delta t\right) .
\end{aligned}
$$

And by induction

$$
\left\|\underline{u}_{T, t}^{0, n}\right\| \leqslant\left(1+2 c_{\max } \Delta t\right)^{n}\left\|\underline{u}_{T, t}^{0}\right\|+\Delta t c_{\max } \sum_{i=1}^{n}\left(1+2 c_{\max } \Delta t\right)^{i} .
$$

Since $\left(1+2 c_{\max } \frac{T}{N}\right)^{N} \leqslant e^{2 T c_{\max }}$ :

$$
\left\|\underline{u}_{T, t}^{0, n}\right\| \leqslant e^{\frac{4}{y_{c}} d_{\min } c_{\max }}\left(\left\|\underline{u}_{T, t}^{0}\right\|+2 c_{\max } \frac{H}{c_{2}} d_{\min }\right) .
$$

Setting

$$
C_{1}^{0}=e^{4 \frac{H}{c_{2}} d_{\min } c_{\max }}\left(\left\|\underline{u}_{T, t}^{0}\right\|+2 \frac{H}{c_{2}} c_{\max } d_{\min }\right),
$$

we deduce immediately

$$
\begin{aligned}
\left\|\underline{b}_{T-}^{0, n+1}\right\| & \leqslant\left\|\underline{b}_{T+}^{0, n+1}\right\|+2\left\|\underline{u}_{T, t}^{0, n}\right\|, \\
& \leqslant B_{T \max }^{0}+2 C_{1}^{0} .
\end{aligned}
$$

Thus

$$
\begin{aligned}
\left\|\underline{u}_{T, t}^{0, n+1}-\underline{u}_{T, t}^{0, n}\right\| & \leqslant \Delta t\left\|F\left(t^{n}+1, \underline{u}_{T, t}^{0, n+1}\right)\right\|, \\
& \leqslant \Delta t c_{\max }\left(1+\left\|\underline{u}_{T, t}^{0, n+1}\right\|\right) \\
& \leqslant \Delta t c_{\max }\left(1+C_{1}^{0}\right)
\end{aligned}
$$

and

$$
\begin{aligned}
\left\|\underline{b}_{T-}^{1, n+1}-\underline{b}_{T-}^{0, n+1}\right\| & \leqslant\left\|\underline{b}_{T-}^{1, n}+d_{1}\left(\underline{b}_{T-}^{0, n}-\underline{b}_{T-}^{1, n}\right)-\underline{b}_{T-}^{0, n+1}\right\| \\
& \leqslant\left(1-d_{1}\right)\left\|\underline{b}_{T-}^{0, n}-\underline{b}_{T-}^{1, n}\right\|+2\left\|\underline{u}_{T, t}^{0, n+1}-\underline{u}_{T, t}^{0, n}\right\|+\left\|\underline{b}_{T+}^{0, n+1}-\underline{b}_{T+}^{0, n}\right\|, \\
& \leqslant\left(1-d_{1}\right) d B_{T \max }^{n}+d_{1} d B_{T \max }^{0}+2 \Delta t c_{\max }\left(1+C_{1}^{0}\right) .
\end{aligned}
$$


And now just as for Proposition 5, we conclude by induction that

$$
d B_{T \max }^{n} \leqslant 2 d B_{T \max }^{0}+2 \frac{\Delta t}{d_{1}} c_{\max }\left(1+C_{1}^{0}\right) .
$$

\subsection{Convergence}

To simplify the proof, we will assume that the ratio $\frac{\Delta t}{\Delta z}$ is constant, i.e. $N_{z}$ is proportional to $N_{t}$ :

$$
N_{z}=\frac{1}{\eta} N_{t}
$$

where $\eta$ is independent of $N_{t}$ and satisfies the CFL condition

$$
\eta \geqslant \frac{c_{2} T}{H}
$$

Let us denote by $\underline{u}_{, t}^{i, n, N_{t}}, \underline{u}_{3}^{i, n, N_{t}}$ the discrete solution given by one of the schemes and $\underline{u}_{t}^{N_{t}}\left(t, x_{3}\right), \underline{u}_{3}^{N_{t}}\left(t, x_{3}\right)$ the linear interpolations of the discrete solutions

$$
\underline{u}_{, t}^{N_{t}}(n \Delta t+\delta t, i \Delta z+\delta z)=(1-\delta t)\left((1-\delta z) \underline{u}_{, t}^{i, n, N_{t}}+\delta z \underline{u}^{i+1, n, N_{t}}\right)+\delta t\left((1-\delta z) \underline{u}_{, t}^{i, n+1, N_{t}}+\delta z \underline{u}^{i+1, n+1, N_{t}}\right),
$$

with $0 \leqslant \delta t \leqslant \Delta t$ and $0 \leqslant \delta z \leqslant \Delta z$. An analogous definition is taken for $\underline{u}_{, 3}^{N_{t}}\left(t, x_{3}\right)$.

Lemma 1. If the sequence $\left(\underline{u}_{, t}^{N_{t}}, \underline{u}_{3}^{N_{t}}\right)_{N_{t}}$ uniformly converges toward a pair of functions $\left(\underline{u}_{,}, \underline{u}_{3}\right)$ and if the functions $\underline{u}_{, t}$ and $\underline{u}_{3}$ are continuous on $[0, T] \times[0, H]$ then these functions satisfy the relations $(18)-(20)$ on the characteristic lines.

Proof. We establish the result on only one relation and one component, the vertical component, because the proof is identical for the other cases. As before, we define

$$
\begin{aligned}
b_{3+}\left(t, x_{3}\right) & =u_{3, t}\left(t, x_{3}\right)+c_{2} u_{3,3}\left(t, x_{3}\right), \\
b_{3+}^{N_{t}}\left(t, x_{3}\right) & =u_{3, t}^{N_{t}}\left(t, x_{3}\right)+c_{2} u_{3,3}^{N_{t}}\left(t, x_{3}\right), \\
b_{3+}^{i, n, N_{t}} & =u_{3, t}^{i, n, N_{t}}+c_{2} u_{3,3}^{i, n, N_{t}} .
\end{aligned}
$$

For any $z_{0}, t_{0}, \delta_{0}$ such that

$$
\delta_{0} \in\left[0, \max \left(T, \frac{H}{c_{2}}\right)\right], \quad z_{0} \in\left[0, H-\eta \delta_{0} \frac{H}{T}\right], \quad t_{0} \in\left[0, T-\delta_{0}\right] .
$$

Let us define

$$
\begin{aligned}
& n_{1}^{N_{t}}=\left\lfloor\frac{N_{t} t_{0}}{T}\right\rfloor, \quad i_{2}^{N_{t}}=\left\lfloor\frac{N_{t} z_{0}}{\eta H}\right\rfloor, \quad n_{2}^{N_{t}}=\left\lfloor\frac{N_{t}\left(t_{0}+\delta_{0}\right)}{T}\right\rfloor, \\
& \Delta_{n}^{N_{t}}=n_{2}^{N_{t}}-n_{1}^{N_{t}},
\end{aligned}
$$

where $\lfloor x\rfloor$ is the integer part of $x$. Of course, $n_{1}^{N_{t}} \Delta t$ converges toward $t_{0}$ when $N_{t}$ goes to infinity; and $\left(n_{2}^{N_{t}} \Delta t, i_{2}^{N_{t}} \Delta z\right)$ converges toward $\left(t_{0}+\delta_{0}, z_{0}\right)$. Thus

$$
b_{3+}^{i_{t}^{N_{t}}, n_{t}^{N_{t}}, N_{t} N_{t} \rightarrow+\infty} \stackrel{b_{3+}}{\longrightarrow}\left(t_{0}+\delta_{0}, 3_{0}\right)
$$


From the Lax-Wendroff scheme, it comes

$$
\begin{aligned}
b_{3+}^{i_{2}^{N_{t}}, n_{2}^{N_{t}}, N_{t}} & =\left(1-d_{2}\right) b_{3+}^{i_{2}^{N_{t}}, n_{2}^{N_{t}}-1, N_{t}}+d_{2} b_{3+}^{i_{2}^{N_{t}}+1, n_{2}^{N_{t}}-1, N_{t}}, \\
& =\left(1-d_{2}\right)^{2} b_{3+}^{i_{t}^{N_{t}}, n_{2}^{N_{t}}-2, N_{t}}+2\left(1-d_{2}\right) d_{2} b_{3+}^{i_{t}^{N_{t}}+1, n_{2}^{N_{t}}-2, N_{t}}+d_{2}^{2} b_{3+}^{i_{2}^{N_{t}}+2, n_{2}^{N_{t}}-2, N_{t}}, \\
& =\cdots \\
& =\sum_{i=0}^{\Delta_{n}^{N_{t}}}\left(\begin{array}{c}
i \\
\Delta_{n}^{N_{t}}
\end{array}\right) d^{i}(1-d)^{\Delta_{n}^{N_{t}}-i} b_{3+}^{i_{t}^{N_{t}}+i, n_{1}^{N_{t}}, N_{t}}, \\
& =\sum_{i=0}^{\Delta_{n}^{N_{t}}} B_{i}^{\Delta_{n}^{N_{t}}}\left(d_{2}\right) b_{3+}^{i_{2}^{N_{t}}+i, n_{1}^{N_{t}}, N_{t}},
\end{aligned}
$$

where $B_{i}^{\Delta_{n}^{N_{t}}}$ is a Bernstein polynomial. With the decomposition

$$
\left.\left.\left.b_{3+}^{i_{2}^{N_{t}}+i, n_{1}^{N_{t}}, N_{t}}=b_{3+} t_{0}, z_{0}+\frac{i}{\Delta_{n}^{N_{t}}}\left(\eta \delta_{0} \frac{H}{T}\right)\right)+b_{3+}^{i_{2}^{N_{t}}+i, n_{1}^{N_{t}}, N_{t}}-b_{3+} t_{0}, z_{0}+\frac{i}{\Delta_{n}^{N_{t}}}\left(\eta \delta_{0} \frac{H}{T}\right)\right)\right),
$$

it comes

$$
\begin{aligned}
b_{3+}^{i_{2}^{N_{t}}, n_{2}^{N_{t}}, N_{t}}= & \sum_{i=0}^{\Delta_{n}^{N_{t}}} B_{i}^{\Delta_{n}^{N_{t}}}\left(d_{2}\right) b_{3+} \\
& \left.t_{0}, z_{0}+\frac{i}{\Delta_{n}^{N_{t}}}\left(\eta \delta_{0} \frac{H}{T}\right)\right) \\
& \left.\left.+\sum_{i=0}^{\Delta_{n}^{N_{t}}} B_{i}^{\Delta_{\Delta_{t}}^{N_{t}}}\left(d_{2}\right) b_{3+}^{N_{t}}\left(n_{1}^{N_{t}} \Delta t,\left(i_{2}^{N_{t}}+i\right) \Delta z\right)-b_{3+} t_{0}, z_{0}+\frac{i}{\Delta_{n}^{N_{t}}}\left(\eta \delta_{0} \frac{H}{T}\right)\right)\right) .
\end{aligned}
$$

From the approximation with Bernstein polynomials, we know that for each continuous function $f:[0,1] \rightarrow \mathbb{R}$, the function $f^{N}=\sum_{i=0}^{N} B_{i}^{N} f\left(\frac{i}{N}\right)$ uniformly converges toward $f$ when $N$ goes to $+\infty$. Since the function $b_{3+}^{N_{t}}$ is continuous, the expression

$$
\left.\sum_{i=0}^{\Delta_{n}^{N_{t}}} B_{i}^{\Delta_{n}^{N_{t}}}\left(d_{2}\right) b_{3+} t_{0}, z_{0}+\frac{i}{\Delta_{n}^{N_{t}}}\left(\eta \delta_{0} \frac{H}{T}\right)\right)
$$

converges towards the value

$$
b_{3+}\left(t_{0}, z_{0}+d_{2}\left(\eta \delta_{0} \frac{H}{T}\right)\right)=b_{3+}\left(t_{0}, z_{0}+\delta_{0} c_{2}\right)
$$

when $\Delta_{n}^{N_{t}}$ goes to $+\infty$.

Now, due to uniform convergence, it comes

$$
\forall \varepsilon, \quad \exists N_{\varepsilon}>0 ; \quad \forall N_{t}>N_{\varepsilon}, \quad \forall(t, z) \in[0, T] \times[0, H],\left|b_{3+}^{N_{t}}(t, z)-b_{3+}(t, z)\right|<\varepsilon .
$$

Considering the uniform continuity of $b_{3+}$ on $[0, T] \times[0, H]$ we get

$$
\forall \varepsilon, \quad \exists \delta_{\varepsilon} ; \quad \forall\left(t_{1}, z_{1}\right), \quad \forall\left(t_{2}, z_{2}\right), \quad d\left(\left(t_{1}, z_{1}\right),\left(t_{2}, z_{2}\right)\right)<\delta_{\varepsilon} \Rightarrow\left|b_{3+}\left(t_{1}, z_{1}\right)-b_{3+}\left(t_{2}, z_{2}\right)\right|<\varepsilon .
$$

And because

$$
\left|t_{0}-n_{1}^{N_{t}} \Delta t\right| \leqslant \Delta t
$$

and

$$
\begin{aligned}
\left|\left(i_{2}^{N_{t}}+i\right) \Delta z-z_{0}+\frac{i}{\Delta_{n}^{N_{t}}}\left(\eta \delta_{0} \frac{H}{T}\right)\right| & \leqslant \Delta z+\Delta z \frac{i}{\Delta_{n}^{N_{t}}}\left(\left\lfloor\frac{t_{0}}{\Delta t}-\left\lfloor\frac{t_{0}}{\Delta t}\right\rfloor+\frac{\delta_{0}}{\Delta t}\right\rfloor-\frac{\delta_{0}}{\Delta t}\right), \\
& \leqslant 3 \Delta z
\end{aligned}
$$


then

$$
\begin{aligned}
\forall \varepsilon, \quad \exists N_{\varepsilon}>0 ; \quad \forall N_{t}>N_{\varepsilon}, \quad & \left|b_{3+}^{N_{t}}\left(n_{1}^{N_{t}} \Delta t,\left(i_{2}^{N_{t}}+i\right) \Delta z\right)-b_{3+}\left(n_{1}^{N_{t}} \Delta t,\left(i_{2}^{N_{t}}+i\right) \Delta z\right)\right|<\varepsilon, \\
& \left|b_{3+}\left(n_{1}^{N_{t}} \Delta t,\left(i_{2}^{N_{t}}+i\right) \Delta z\right)-b_{3+}\left(t_{0}, z_{0}+\delta_{0} c_{2}\right)\right|<\varepsilon .
\end{aligned}
$$

and

$$
\forall \varepsilon, \quad \exists N_{\varepsilon}>0 ; \quad \forall N_{t}>N_{\varepsilon}, \quad\left|b_{3+}^{N_{t}}\left(n_{1}^{N_{t}} \Delta t,\left(i_{2}^{N_{t}}+i\right) \Delta z\right)-b_{3+}\left(t_{0}, z_{0}+\delta_{0} c_{2}\right)\right|<2 \varepsilon .
$$

then the expression

$$
\left.\left.\sum_{i=0}^{\Delta_{n}^{N_{t}}} B_{i}^{\Delta_{n}^{N_{t}}}\left(d_{2}\right) \quad b_{3+}^{N_{t}}\left(n_{1}^{N_{t}} \Delta t,\left(i_{2}^{N_{t}}+i\right) \Delta z\right)-b_{3+} t_{0}, z_{0}+\frac{i}{\Delta_{n}^{N_{t}}}\left(\eta \delta_{0} \frac{H}{T}\right)\right)\right),
$$

converges to zero when $N_{t}$ goes to $+\infty$.

We just established that the relation

$$
b_{3+}\left(t_{0}+\delta_{0}, z_{0}\right)=b_{3+}\left(t_{0}, z_{0}+c_{2} \delta_{0}\right),
$$

is satisfied for all $\delta_{0} \in\left[0, \max \left(T, \frac{H}{c_{2}}\right)\right]$, and for all pairs $\left(t_{0}, z_{0}\right)$ satisfying

$$
z_{0} \in\left[0, H-\eta \delta_{0} \frac{H}{T}\right], \quad t_{0} \in\left[0, T-\delta_{0}\right] .
$$

It is not sufficient to conclude, because we need to establish the result for all pairs $\left(t_{0}, z_{0}\right)$ satisfying

$$
z_{0} \in\left[0, H-c_{2} \delta_{0}\right], \quad t_{0} \in\left[0, T-\delta_{0}\right] .
$$

But, equality (64) can be split in two parts:

$$
\begin{aligned}
b_{3+}\left(t_{0}+\frac{\delta_{0}}{2}, z_{0}+c_{2} \frac{\delta_{0}}{2}\right) & =b_{3+}\left(t_{0}, z_{0}+c_{2} \frac{\delta_{0}}{2}\right), \\
b_{3+}\left(t_{0}+\delta_{0}, z_{0}\right) & =b_{3+}\left(t_{0}+\frac{\delta_{0}}{2}, z_{0}+c_{2} \frac{\delta_{0}}{2}\right) .
\end{aligned}
$$

which are established for

$$
z_{0} \in\left[0, H-c_{2} \frac{\delta_{0}}{2}-\eta \frac{\delta_{0}}{2} \frac{H}{T}\right]
$$

Using this reasoning, one sees that relation (64) is satisfied for

$$
z_{0} \in\left[0, H-c_{2} \frac{\delta_{0}}{2}[.\right.
$$

And since the function $b_{3+}$ is continuous, this relation is also satisfied for

$$
z_{0} \in\left[0, H-c_{2} \frac{\delta_{0}}{2}\right]
$$

Proposition 7. If the following hypotheses are satisfied:

- the initial data $\underline{u}_{0}$ is differentiable and is derivative is Lipschitz continuous,

- the initial data $\underline{u}_{1}$ is Lipschitz continuous,

- the ratio $\eta=\frac{N_{t}}{N_{z}}$ is constant and satisfies $\eta \geqslant \frac{c_{2} T}{H}$,

then schemes (39)-(46), (49)-(52) is convergent.

Proof. Let us still consider $\underline{u}_{t}^{N_{t}}\left(t, x_{3}\right), \underline{u}_{3}^{N_{t}}\left(t, x_{3}\right)$ the linear interpolations of the discrete solutions of schemes (39)-(46), (49)-(52). From Propositions 5 and 6 these functions are bounded in $\left(L^{\infty}([0, T] \times[0, H])\right)^{3}$ 
independently of $N_{t}$, and moreover exist $C_{3}$ and $C_{4}$, two positive constants which only depend on the initial data such that

$$
\begin{aligned}
& \max _{0 \leqslant i \leqslant N_{z}-1}\left(\left\|\underline{u}_{, t}^{i+1, n, N_{t}}-\underline{u}_{, t}^{i, n, N_{t}}\right\|,\left\|\mathbf{C} \underline{u}_{3}^{i+1, n, N_{t}}-\mathbf{C} \underline{u}_{,}^{i, n, N_{t}}\right\|\right) \\
& \quad<C_{3} \max _{0 \leqslant i \leqslant N_{z}-1}\left(\left\|\underline{u}_{1}((i+1) \Delta t)-\underline{u}_{1}(i \Delta t)\right\|,\left\|\mathbf{C} \partial_{x_{3}} \underline{u}_{0}((i+1) \Delta t)-\mathbf{C} \partial_{x_{3}} \underline{u}_{0}(i \Delta t)\right\|\right)+C_{4} \Delta t .
\end{aligned}
$$

Since we assume $\underline{u}_{0}$ is differentiable and is derivative is Lipschitz and $\underline{u}_{1}$ is Lipschitz, we conclude that $\underline{u}_{t}^{N_{t}}$ and $\underline{u}_{3}^{N_{t}}$ are bounded in $\left(W^{1, \infty}(] 0, T[\times] 0, H[)\right)^{3}$ independently of $N_{t}$.

From the Rellich-Kondrachov Theorem (see [1] for instance) the space $W^{1, \infty}(] 0, T[\times] 0, H[)$ is included in the space $\mathscr{C}([0, T] \times[0, H])$ of the continuous functions on $[0, T] \times[0, H]$, and the inclusion is compact. Thus, from the sequence $\left(\underline{u}_{, t}^{N_{t}}, \underline{u}_{3}^{N_{t}}\right)_{N_{t}}$ it is possible to extract a sub-sequence $\left(\underline{u}_{, t}^{N_{t}}, \underline{u}_{3}^{N_{t}}\right)_{N_{t}^{\prime}}$ which uniformly converges toward a pair of continuous functions $\left(\underline{u}_{t}, \underline{u}_{3}\right)$.

From Lemma 1, we know that $\underline{u}_{, t}\left(t, x_{3}\right)$ and $\underline{u}_{3}\left(t, x_{3}\right)$ satisfy to the relations (18)-(20) on the characteristic lines. In particular, for $t<\frac{H}{c_{2}}$, we get

$$
\begin{aligned}
& b_{1+}(t, 0)=u_{11}\left(c_{1} t\right)+c_{1} \partial_{x_{3}} u_{10}\left(c_{1} t\right), \\
& b_{2+}(t, 0)=u_{21}\left(c_{1} t\right)+c_{1} \partial_{x_{3}} u_{20}\left(c_{1} t\right), \\
& b_{3+}(t, 0)=u_{31}\left(c_{2} t\right)+c_{2} \partial_{x_{3}} u_{30}\left(c_{2} t\right) .
\end{aligned}
$$

And, because of the uniform convergence, the functions $b_{1+}^{N_{t}^{\prime}}(., 0), b_{2+}^{N_{t}^{\prime}}(., 0)$ and $b_{3+}^{N_{t}^{\prime}}(., 0)$ uniformly converge toward the respective values $u_{11}\left(c_{1} t\right)+c_{1} \partial_{x_{3}} u_{10}\left(c_{1} t\right), u_{21}\left(c_{1} t\right)+c_{1} \partial_{x_{3}} u_{20}\left(c_{1} t\right)$ and $u_{31}\left(c_{2} t\right)+c_{2} \partial_{x_{3}} u_{30}\left(c_{2} t\right)$.

The expression of the implicit Euler scheme is

$$
\begin{aligned}
u_{3}^{0, n+1, N_{t}^{\prime}}= & \left(u_{3}^{0, n, N_{t}^{\prime}}+\Delta t b_{3+}^{0, n+1, N_{t}^{\prime}}\right)_{+}+\frac{1}{1+\frac{\Delta t}{\tilde{\eta}}}\left(u_{3}^{0, n, N_{t}^{\prime}}+\Delta t b_{3+}^{0, n+1, N_{t}^{\prime}}\right)_{-}, \\
S^{n+1, N_{t}^{\prime}}= & -\frac{\lambda+2 G}{c_{2}}\left(b_{3+}^{0, n+1, N_{t}^{\prime}}-u_{3, t}^{0, n+1, N_{t}^{\prime}}\right) \\
\underline{u}_{T, t}^{0, n+1, N_{t}^{\prime}} \in & \underline{u}_{T, t}^{0, n, N_{t}^{\prime}}+\frac{\Delta t}{\varepsilon}\left(\frac{G}{c_{1}}\left(b_{T+}^{0, n+1, N_{t}^{\prime}}-\underline{u}_{T, t}^{0, n+1, N_{t}^{\prime}}\right)\right. \\
& \left.-S^{n+1, N_{t}^{\prime}} \mu\left(\left\|\underline{u}_{T, t}^{0, n+1, N_{t}^{\prime}}-\underline{V}_{e}\left(t^{n+1}\right)\right\|\right) \operatorname{Dir}\left(\underline{u}_{T, t}^{0, n+1, N_{t}^{\prime}}-\underline{V}_{e}\left(t^{n+1}\right)\right)\right),
\end{aligned}
$$

which can be rewritten as

$$
\begin{aligned}
u_{3}^{0, n+1, N_{t}^{\prime}}= & \left(u_{3}^{0, n, N_{t}^{\prime}}+\Delta t b_{3+}\left(t^{n+1}, 0\right)\right)_{+}+\frac{1}{1+\frac{\Delta t}{\tilde{\eta}}}\left(u_{3}^{0, n, N_{t}^{\prime}}+\Delta t b_{3+}\left(t^{n+1}, 0\right)\right)_{-}+\epsilon_{3}^{n+1, N_{t}^{\prime}}, \\
S^{n+1, N_{t}^{\prime}}= & -\frac{\lambda+2 G}{c_{2}}\left(b_{3+}\left(t^{n+1}, 0\right)-u_{3, t}^{0, n+1, N_{t}^{\prime}}\right) \\
\underline{u}_{T, t}^{0, n+1, N_{t}^{\prime}} \in & \underline{u}_{T, t}^{0, n, N_{t}^{\prime}}+\frac{\Delta t}{\varepsilon}\left(\frac{G}{c_{1}}\left(b_{T+}\left(t^{n+1}, 0\right)-\underline{u}_{T, t}^{0, n+1, N_{t}^{\prime}}\right)\right. \\
& \left.-S^{n+1, N_{t}^{\prime}} \mu\left(\left\|\underline{u}_{T, t}^{0, n+1, N_{t}^{\prime}}-\underline{V}_{e}\left(t^{n+1}\right)\right\|\right) \underline{\operatorname{Dir}}\left(\underline{u}_{T, t}^{0, n+1, N_{t}^{\prime}}-\underline{V}_{e}\left(t^{n+1}\right)\right)\right)+\epsilon_{T}^{n+1, N_{t}^{\prime}} .
\end{aligned}
$$

This scheme is an implicit Euler scheme for the Cauchy problem corresponding to Eq. (38) and the Cauchy problem (35). Now, under the conditions that the right-hand side of the differential inclusion of (35) is onesided Lipschitz and satisfies the growth condition (63) and also that the quantities $\epsilon_{3}^{n+1, N_{t}^{\prime}}$, and $\epsilon_{T}^{n+1, N_{t}^{\prime}}$ satisfy

$$
\lim _{N_{t} \rightarrow \infty}\left[\max _{1 \leqslant j \leqslant N}\left(\left\|\epsilon_{T}^{n, N_{t}^{\prime}}\right\|,\left|\epsilon_{3}^{n, N_{t}^{\prime}}\right|\right)\right]=0
$$

it is a result of Lempio (see $[9,6]$ ) that this scheme is convergent. The quantities $\epsilon_{3}^{n+1, N_{t}^{\prime}}$, and $\epsilon_{T}^{n+1, N_{t}^{\prime}}$ satisfy these conditions because the following estimations hold: 


$$
\begin{aligned}
\left|\epsilon_{3}^{n, N_{t}^{\prime}}\right| & \leqslant\left(\frac{1}{1+\frac{\Delta t}{\tilde{\eta}}}\right)\left|b_{3+}\left(t^{n+1}, 0\right)-b_{3+}^{0, n+1, N_{t}^{\prime}}\right|, \\
& \leqslant 2 \Delta t\left|b_{3+}\left(t^{n+1}, 0\right)-b_{3+}^{0, n+1, N_{t}^{\prime}}\right| \\
\left\|\epsilon_{T}^{n, N_{t}^{\prime}}\right\| & \leqslant \frac{\Delta t}{\varepsilon}\left(\frac{G}{c_{1}}+\frac{\lambda+2 G}{c_{2}}\|\mu\|_{\infty}\right)\left\|b_{T+}\left(t^{n+1}, 0\right)-b_{T+}^{0, n+1, N_{t}^{\prime}}\right\|,
\end{aligned}
$$

and because the functions $b_{T+}^{N_{t}^{\prime}}(., 0)$ and $b_{T+}^{N_{t}^{\prime}}(., 0)$ uniformly converge, respectively toward $b_{T+}(., 0)$ and $b_{3+}(., 0)$.

In conclusion, for $t \in\left[0, \frac{H}{c_{2}}\right]$ the function $u(t, 0)_{3, t}$ is the derivative with respect to time of the unique solution of the Cauchy problem associated to the differential equation (38) and the function $u(t, 0)_{T, t}$ is the unique solution of (35). Additionally, From Lemma (1), the functions $\underline{u}_{t}\left(t, x_{3}\right)$ and $\underline{u}_{3}\left(t, x_{3}\right)$ satisfy to the relations 18,18,20 on the characteristic lines. From the uniqueness of this solution, we can conclude that all the uniformly convergent sub-sequences of the sequence $\left(\underline{u}_{t}^{N_{t}}, \underline{u}_{3}^{N_{t}}\right)_{N_{t}}$ converge toward $\left(\underline{u}_{, t}, \underline{u}_{3}\right)$. This is sufficient to say that the whole sequence $\left(\underline{u}_{, t}^{N_{t}}, \underline{u}_{, 3}^{N_{t}}\right)_{N_{t}}$ uniformly converges toward $\left(\underline{u}_{,}, \underline{u}_{, 3}\right)$.

\section{Numerical experiments}

In this section, we present a few numerical experiments made with scheme (39)-(48), 51,52, i.e. with the non-regularized unilateral contact condition. Mechanical parameters for the material corresponds to a little metallic piece $\left(\lambda=107 \times 10^{9} \mathrm{~Pa}, G=77 \times 10^{9} \mathrm{~Pa}, \rho=7800 \mathrm{Kg} / \mathrm{m}^{3}\right)$ of height $5 \mathrm{~cm}$. The coefficient of friction has the shape of Fig. 2, with $\mu_{\mathrm{s}}=0.8$ and $\mu_{\mathrm{d}}=0.5$.

\subsection{Numerical test of convergence}

We here test the stability and the convergence of schemes (39)-(48), 51 on an example. This convergence has not been established theoretically. In order to have a relevant case, we choose an example where the contact pressure varies, and where sometimes the contact does not hold. The perturbation parameter for the friction condition is chosen constant $(\varepsilon=10.0)$. The computations are made with different values of $\Delta t$ and $\Delta z$ with a constant ratio $\frac{\Delta t}{\Delta z}$.

The results of computations are presented on Figs. 3 and 4.

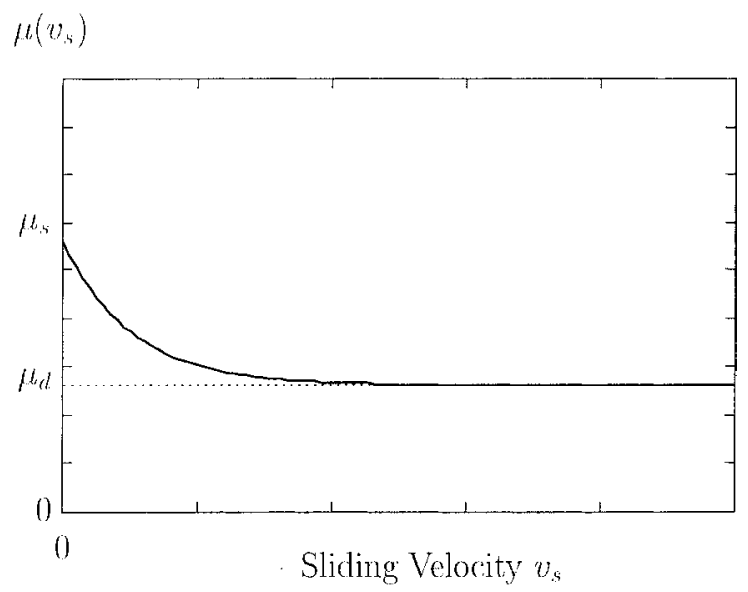

Fig. 2. Example of friction coefficient. 


\begin{tabular}{|c|c|c|c|c|}
\hline Expcriment & $\Delta t$ & $\Delta z$ & $\left\|v_{x}^{n}-v_{x}^{n-1}\right\|_{\infty}$ & $\left\|u_{z}^{n}-u_{z}^{n-1}\right\|_{\infty}$ \\
\hline 1 & $10^{-7}$ & $10^{-4}$ & & \\
\hline 2 & $4 \times 10^{-8}$ & $4 \times 10^{-5}$ & 0.3678 & $2.5342 \times 10^{-6}$ \\
\hline 3 & $10^{-8}$ & $10^{-5}$ & 0.1277 & $9.2298 \times 10^{-7}$ \\
\hline 4 & $4 \times 10^{-9}$ & $4 \times 10^{-6}$ & 0.0905 & $7.1579 \times 10^{-7}$ \\
\hline 5 & $10^{-9}$ & $10^{-6}$ & 0.0237 & $2.8334 \times 10^{-7}$ \\
\hline
\end{tabular}

Fig. 3. Different values of $\Delta t, \Delta z$ and maximal difference between two successive computations.
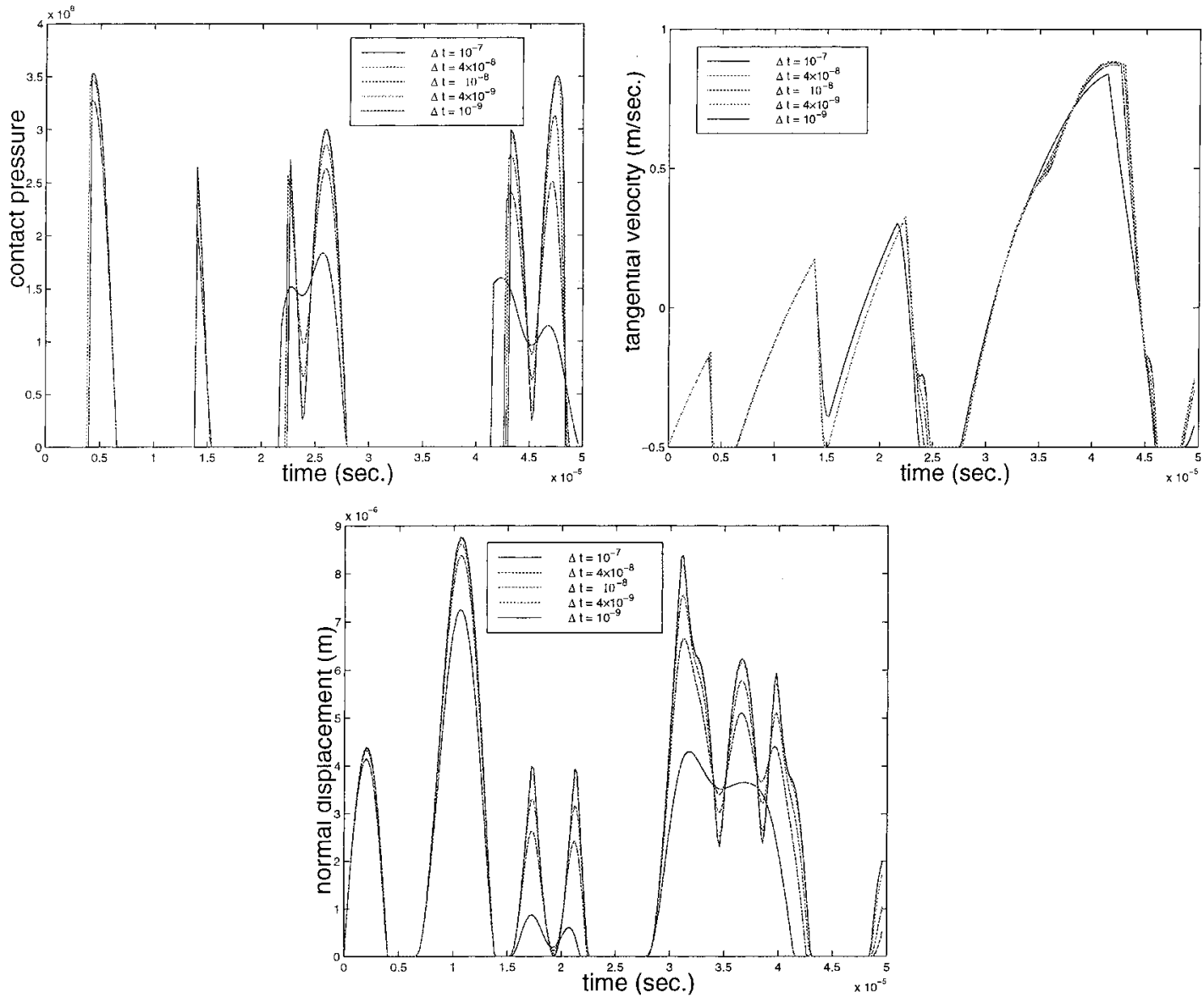

Fig. 4. Evolution of contact pressure, tangential velocity and normal displacement.

There is a numerical convergence, but, due to the bad regularity of the continuous problem solution, the rate of convergence seems to be lower than linear on $\Delta t$.

\subsection{Example of global behavior}

Now, we choose a small perturbation parameter $(\varepsilon=0.1)$ and a constant contact pressure.

Fig. 5 presents the evolution of tangential displacement on the contact boundary. One can see that there is a chaotic behavior at the beginning of the simulation, but, after a certain time of simulation the motion becomes more regular. Fig. 6 presents a detail of the simulation where the motion is regular. This dynamical system seems to be attracted by a solution which is nearly periodical. It is a classical result that a one degree of freedom elastic system with such a law of friction often has a periodic attractor. Here, for the infinite slab, the difference is the infinite number of degree of freedom and the presence of jump in velocity. 

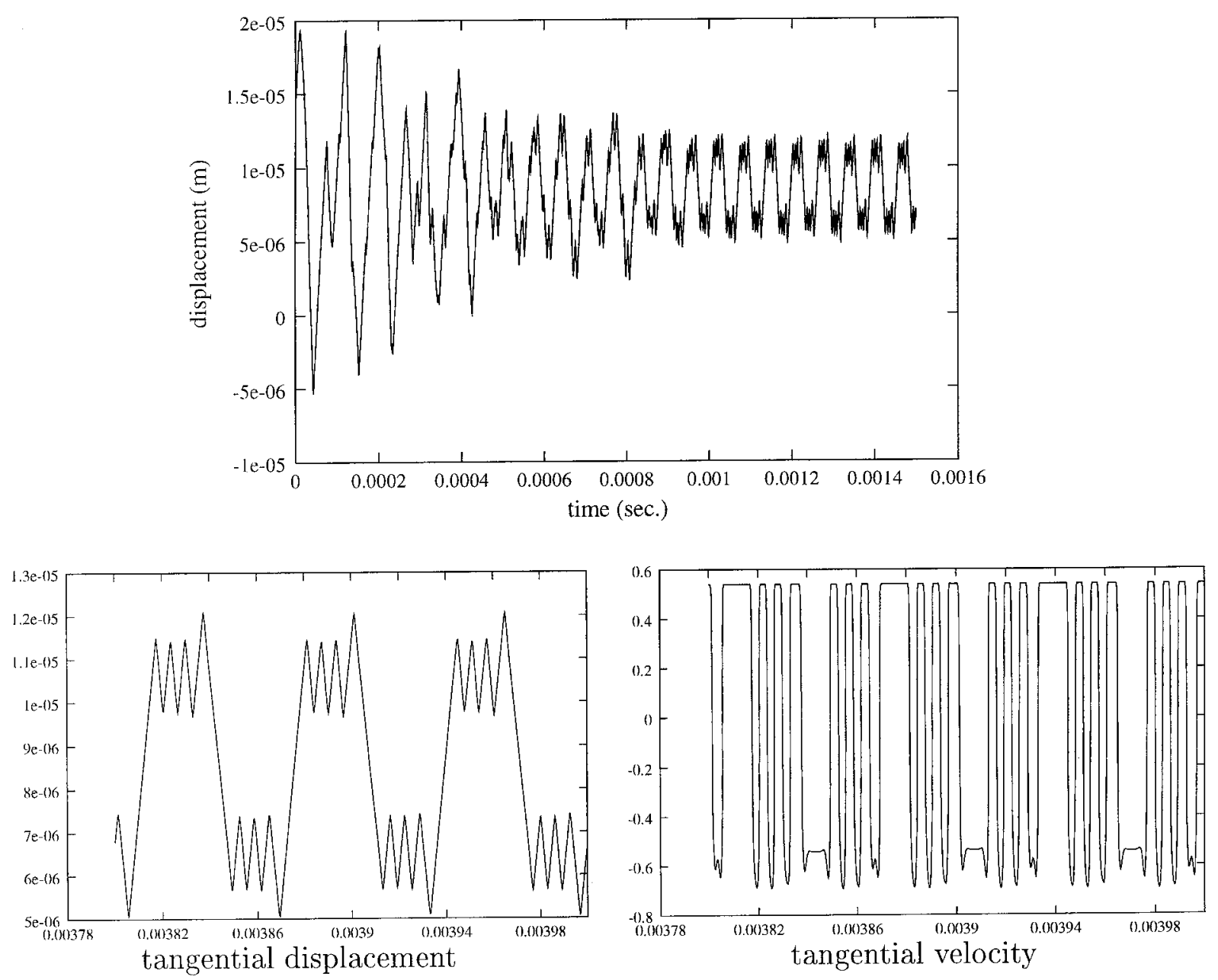

Fig. 5. Evolution of the tangential displacement on the contact boundary.

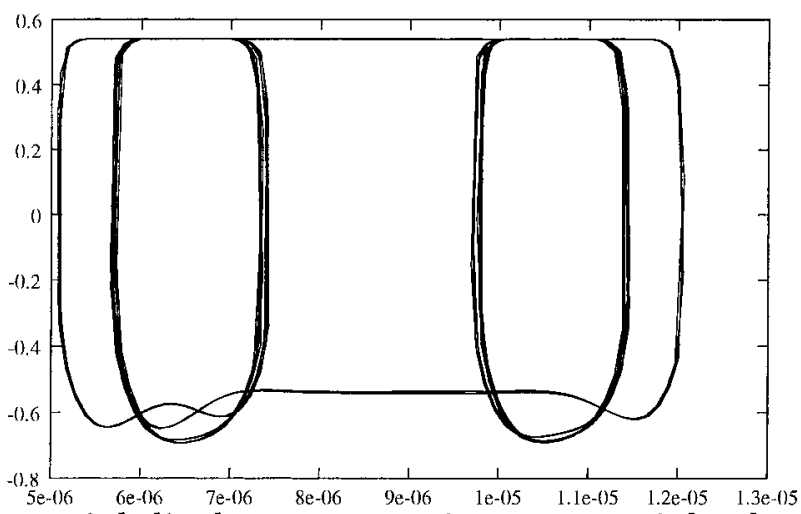

tangential displacement against tangential velocity

Fig. 6. Detail of the evolution of tangential displacement and velocity. 


\section{References}

[1] R.A. Adams, Sobolev Spaces, Academic Press, New York, 1975.

[2] F.P. Bowden, D. Tabor, Friction lubrification and wear: A survey of work during the last decade, Brit. J. Appl. Phys. 17 (1966) $1521-1544$.

[3] M. Comninou, J. Dundurs, Elastic interface waves and sliding between two solids, J. Appl. Mech. 45 (1978) $325-330$.

[4] K. Deimling, Multivalued differential equations, Walter de Gruyter Berlin, 1992.

[5] J.H. Dieterich, Time-dependent friction and the mechanics of stick-slip, Pure \& Appl. Geophys., 116 (1978) $790-806$.

[6] A. Dontchev, F. Lempio, Difference methods for differential inclusions: a survey, SIAM Rev. 34 (2) (1992) $263-294$.

[7] C. Gao, D. Kuhlmann-Wilsdorf, On stick-slip and velocity dependence of friction at low speeds, ASME J. Tribology, 112 (1990) $355-360$.

[8] I.R. Ionescu, J.-C. Paumier, On the contact problem with slip rate dependent friction in elastodynamic, Euro. J. Mech. A/Solids, 4 (1994) 555-568.

[9] F. Lempio, Modified Euler methods for differential inclusions, in: Proceedings of the IIASA Workshop on Set-Valued Analysis and Differential Inclusions, Pamporovo, Bulgaria, 17-21 September 1990.

[10] A.I. Leonov, A. Srinivasan, Self-oscillations of an elastic plate sliding over a smooth surface, Int. J. Eng. Sci. 31 ( 3) (1993) 453-473.

[11] J.A.C. Martins, F.M.F. Simões, On some Sources of Instabilities/Ill-posedness in Elasticity Problems with Coulomb Friction, Contact Mechanics, in: M. Raous, M. Jean, J.J. Moreau (Eds.), Proceedings of the Second Contact Mechanics International Symposium September 19-23 in Carry-Le-Rouet France, 1994, pp. 95-106.

[12] J.T. Oden, J.A.C. Martins, Models and computational methods for dynamic friction phenomena, Comp. Meth. Appl. Mech. Engrg. 52 (1985) 527-634.

[13] J.-C. Paumier, Y. Renard, Frottement Elastodynamique avec Inertie de Surface Rapport IMAG RT 179 Université Joseph Fourier, Grenoble, France, 1999.

[14] E. Rabinowicz, The intrinsic variables affecting the stick-slip process, in: Proceedings of the Royal Physic Society, 71 (1958) $668-675$.

[15] M. Raous, J. Martins, S. Barbarin, Caractérisation numérique d'instabilités dues au frottement: ondes de contraintes dans un contact glissant, 26éme congrés d'analyse numérique, Savoie, 1994, C32-C33.

[16] M. Raous, S. Barbarin, Stress waves in a sliding contact, Part 2: modeling The Third Body Concept, Elsevier, Amsterdam, 1996, $39-44$.

[17] Y. Renard, Dynamic dry friction with a slip velocity dependent coefficient, in: Proceedings of the Third Summer Conference on Numerical Modelling in Continuum Mechanics Prague Part II, 1997, 426-433.

[18] Y. Renard, Modélisation des instabilités liées au frottement sec des solides élastiques aspects théoriques et numériques, thèse de doctorat LMC-IMAG Grenoble, 1998.

[19] Y. Renard, Singular perturbation approach to an elastic dry friction problem with a non-monotone friction coefficient, to appear in Quarterly of applied Mathematics, 1998.

[20] A. Ruina, Slip instability and state variable friction laws, J. Geoph. Res. 88 (B12) (1983) 10359-10370.

[21] D.M. Tolstoi, Significance of the normal degree of freedom and natural vibrations in contact with friction, Wear 10 (1967) 199-213. 